\title{
Optimization and Simulation of Sorting Strategy for Distribution Center
}

\author{
Li Teng ${ }^{1, a}$ \\ ${ }^{1}$ Harbin University of Commerce, Harbin, China \\ aliteng_ha@126.com
}

Keywords: Sorting Strategy. EIQ. Flexsim. Distribution Center.

\begin{abstract}
Sorting operation is the key link for the distribution center operation process. Purpose of sorting is setting customers' ordered goods correctly and quickly, so with which the efficient and feasible sorting strategy is the prerequisite of sorting efficiency. In this paper, optimization and simulation of sorting strategy are studied, in which sorting efficiency is considered as key factor, EIQ analysis is used for proposing optimized strategy and then optimized strategies are simulated and verified by Flexsim model.
\end{abstract}

\section{Introduction}

Sorting strategy determines the efficiency and the ultimate effect of the sorting operation. Core process of distribution operation is sorting operation, the proportion of sorting operation in distribution center operations is growing, and in the same time, it is the most labor-intensive and time-consuming job, and which is the key factor to evaluate the level of distribution service. How could keep the lower sorting error rate with the right goods, right quality, and delivery to customers at the right time is the ultimate purpose for sorting operation.

Normal sorting strategy can be divided into four categories: partition, order division, order batching and order classification. The four strategies can be used either alone, or be used association with each other. Many domestic and international scholars carried on a great deal of research on this ${ }^{[1-4]}$, like sorting-to-order, hybrid sorting. Pushpa Rani and Sudesh Rani study a new parallel sorting algorithm based on partitioning and redistribution, Rajesh Ramachandran and E. Kirubakaran did a comparative study on Kakkot sort and other sorting methods, Shamim Akhter provided new adaptive sorting approach. Orders which is one of the driving factors for sorting operation in distribution center, must be fully analyzed. Entry-Item-Quantity (EIQ) analysis method, which based on order entry, item and quantity, is an effective method of analyzing orders ${ }^{[5]}$. Another auxiliary method for strategy research is based on simulation, which is proven to be effective and intuitive to carry out a test of the proposed strategies ${ }^{[6,7]}$.

This paper study of optimization and simulation of sorting strategy in distribution center by EIQ analysis method and flexsim model, which in order to get the general optimizing sorting strategy method. The question of sorting operation in distribution center is first described in section 2, and then optimization strategy based on EIQ analysis and simulation results based on Flexsim are addressed in section 3 and 4 .

\section{Question Description}

A distribution center has three major type of goods, the quality and number of each item are shown as Figure 1. From the chart, demands for item I are the most, followed by item III, which has basically the same demands with item I, and item II has the minimum demands. Different demands are means of different handing numbers, and also affect sorting cost and efficiency. So the sorting-to-order with partition strategy is currently adopted in the distribution center, after partition, handing distance of item I and item III are efficiently reduced.

Following are the details. The item I,II and III are divided into A, B, and C three areas, the shelves of two kinds of large shipments, that is item I and item III, are placed in front of item IIs'. Item I and 
item III are located in area A and B, and item II are located in area C, then every order is sorted. The process is shown in Figure 2.

proportion of IQ

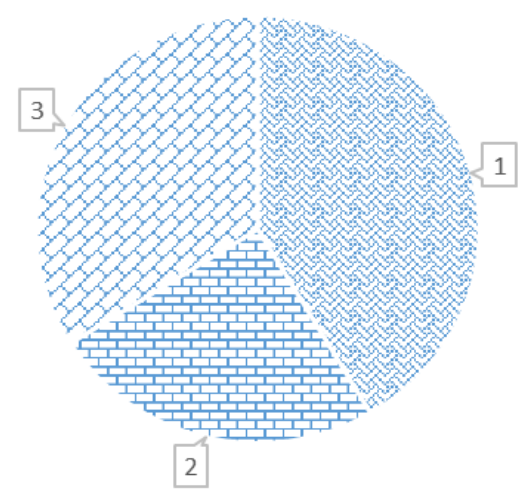

proportion of IK

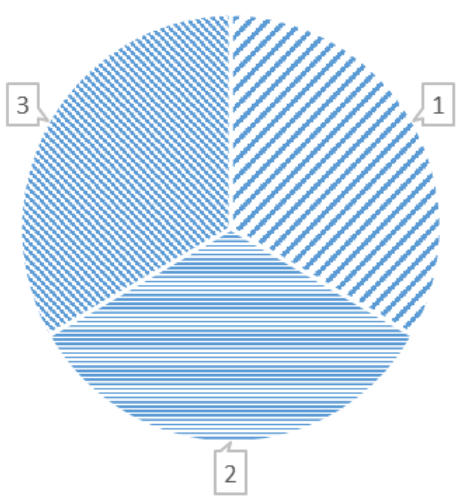

Figure 1. Proportion chart of IQ and IK

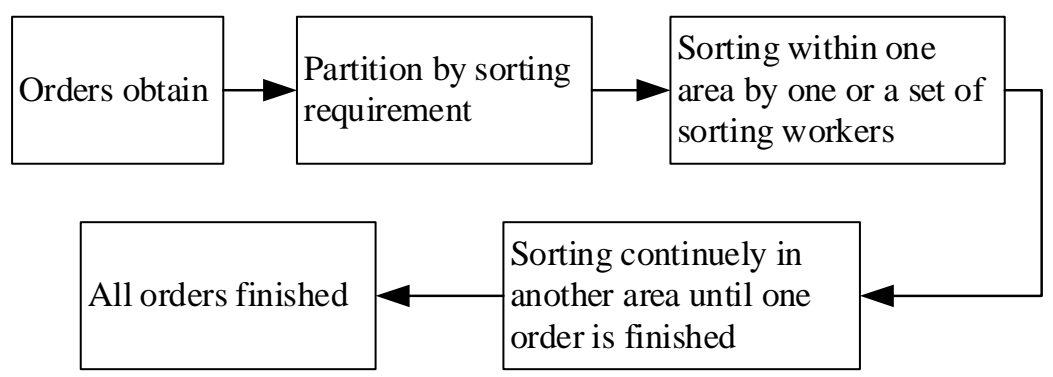

Figure 2. Sorting Process with sorting-to-order strategy

The enterprise that this distribution center served changing its marketing pattern to direct marketing mode which makes orders becoming much frequency and little batch, the changes of order can be seen in Table 1. So the sorting strategy should be changed. Figure 3 shows the enterprise's marketing pattern, in the figure, the value of parameters $\mathrm{k}$, $\mathrm{j}$ are significantly less than parameters $\mathrm{m}$ and $n$.

Table 1 Comparison of order's change

\begin{tabular}{ccc}
\hline & order before change(one day) & order after change(one day) \\
\hline order entry & 15 & 86 \\
EN & 3 & 3 \\
EQ(average) & 143 & 52 \\
EQ(max) & 210 & 70 \\
EQ(min) & 115 & 35 \\
\hline
\end{tabular}

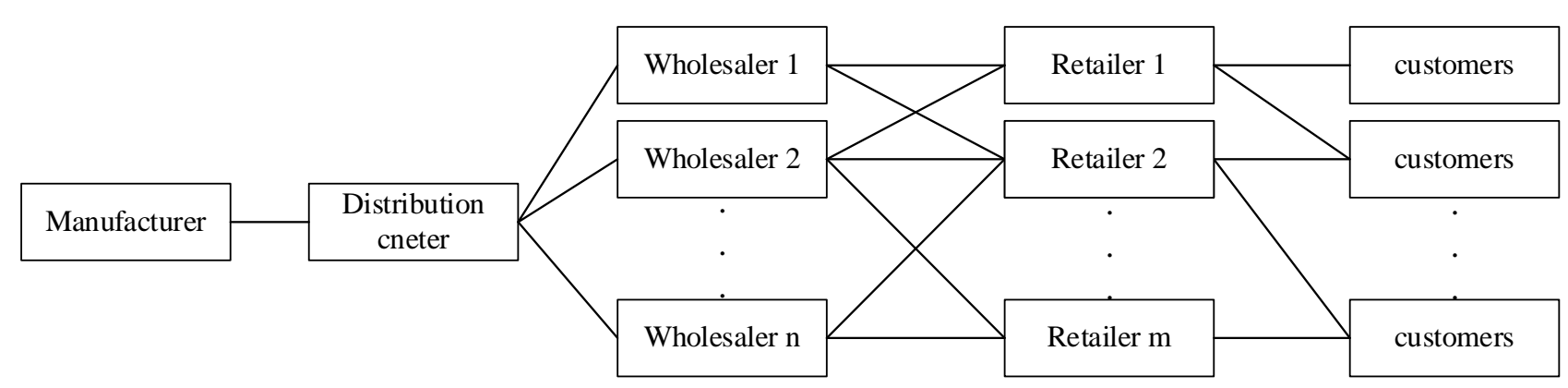

a) The enterprise's older marketing pattern 


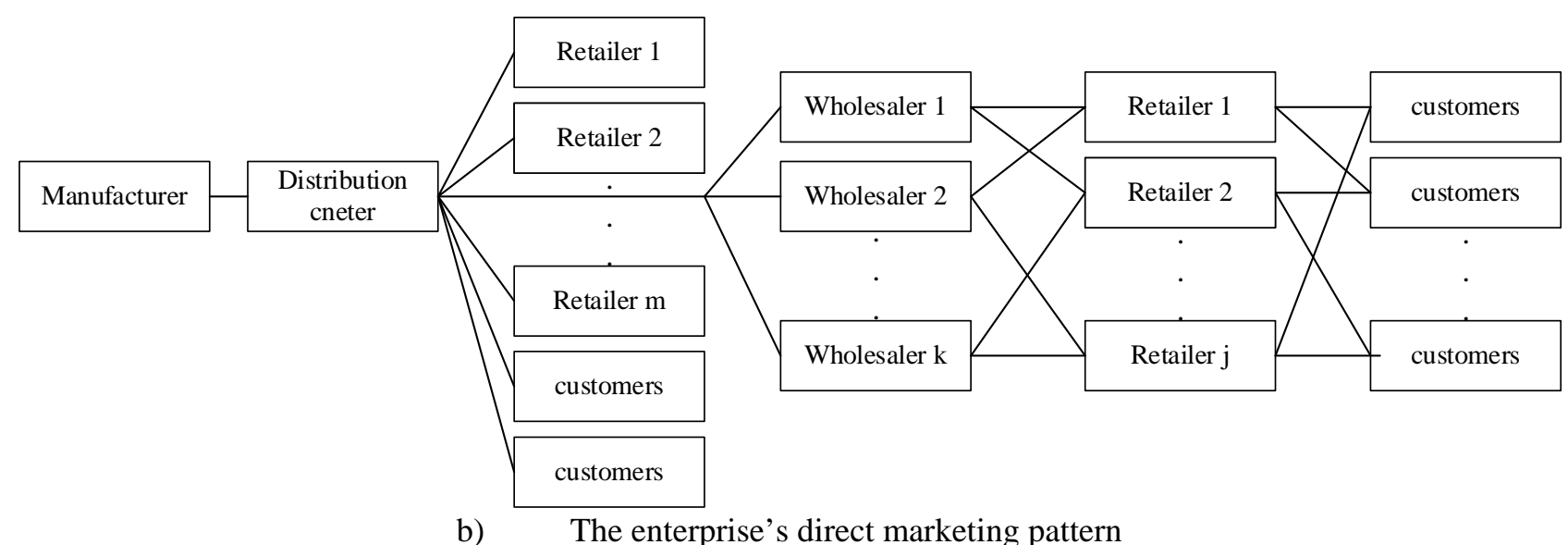

Figure 3. The enterprise's marketing pattern

\section{Optimization Strategy}

The optimization flow chart is firstly shown in Figure 4.
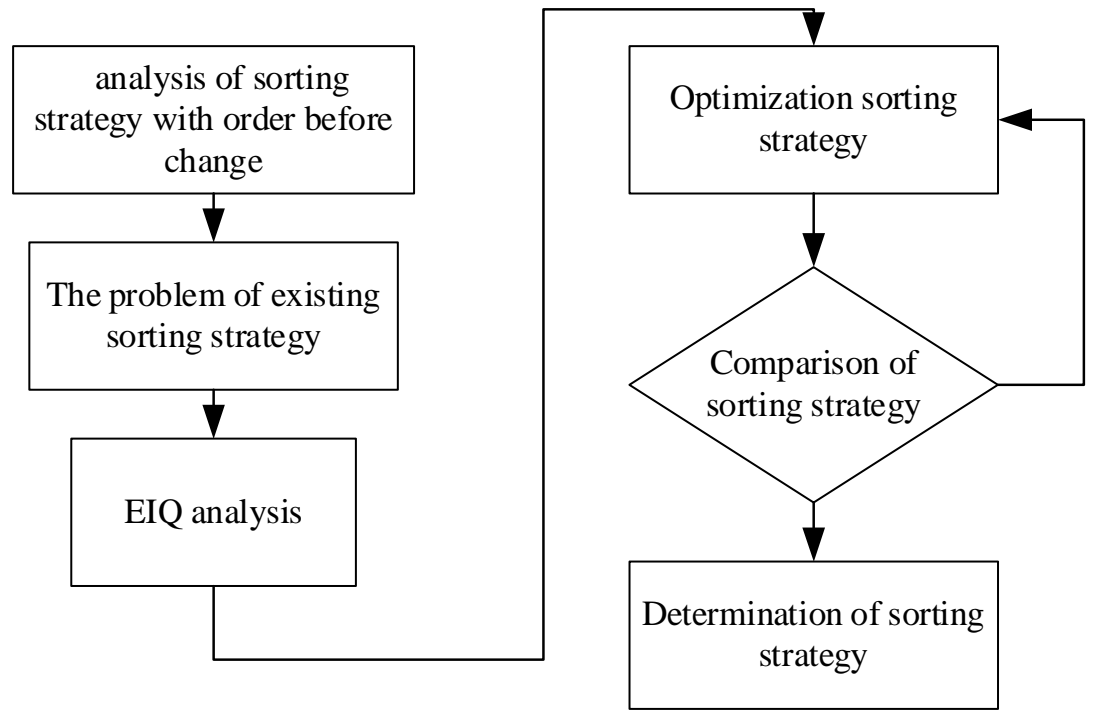

Figure 4. Optimization flow chart

A simple analysis of EQ, EN, IQ, IK is done using EIQ data, and the IQ chart diagram is shown in Figure 5. From the figure, customer order have a relatively large demand on item I and item III, similar to the original situation, so the partition strategy is also adopted with the shelves of two kinds of large shipments place in the forward.

\section{proportion of IQ}

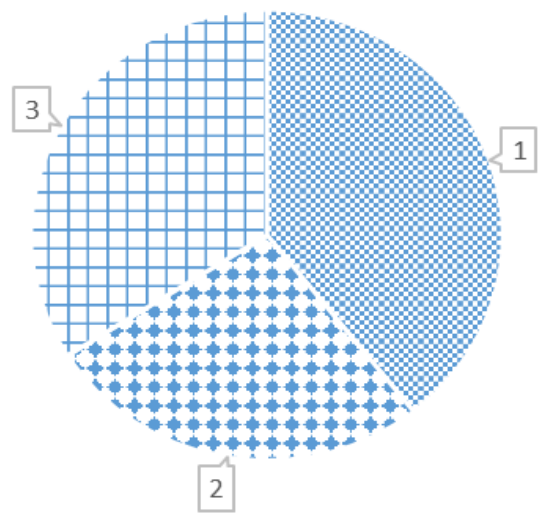

Figure 5. Proportion chart of IQ for new orders 
Then the comparison EQ chart with order items before and after change are drawn in Figure 6. In the figure, solid line shows the EQ data with order before change, and the dotted line shows the EQ data with order after change. From EQ chart, the EQ variation trend of order before change is basically consistent with that of order after change, with conform to the laws: most of order item's quality are going to be close to the average value, a small number of order items with smaller or larger qualities. The quality of orders' items after change is decreased by one third to the original one with order quality increased 5.7 times, so the sorting operation has the heavier workload then before. In order to improve sorting efficiency, batch sorting strategy is choosen according to the combined measurement in batche.

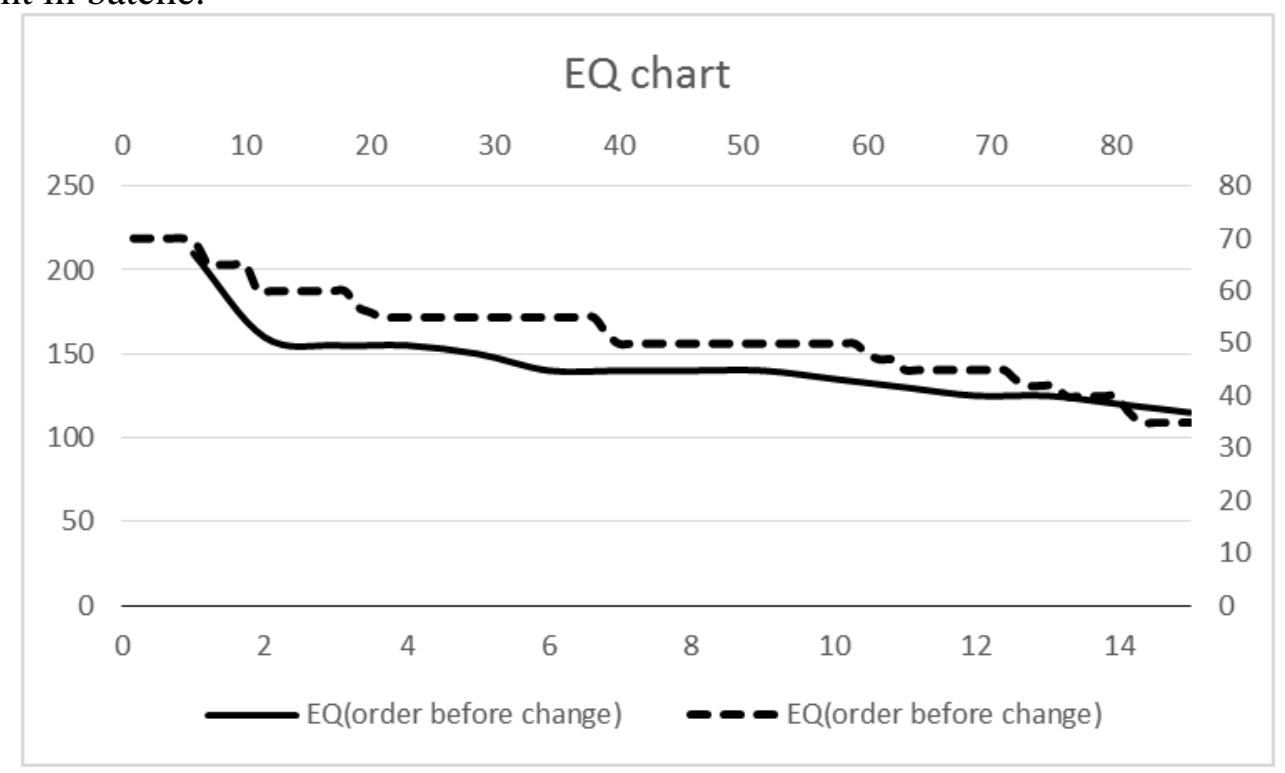

Figure 6. Comparison EQ chart before and after change

The average quality of order items in this distribution center is about 4400 pieces, generally speaking, if shipment of one distribution center is below 5000 pieces, manual sorting eith certain logistics equipments are suitable.

The comparison IK chart with order items before and after change are drawn in Figure 7. From the figure, almost all orders will contain all kinds of items. So the batch sorting strategy is an suitable way.

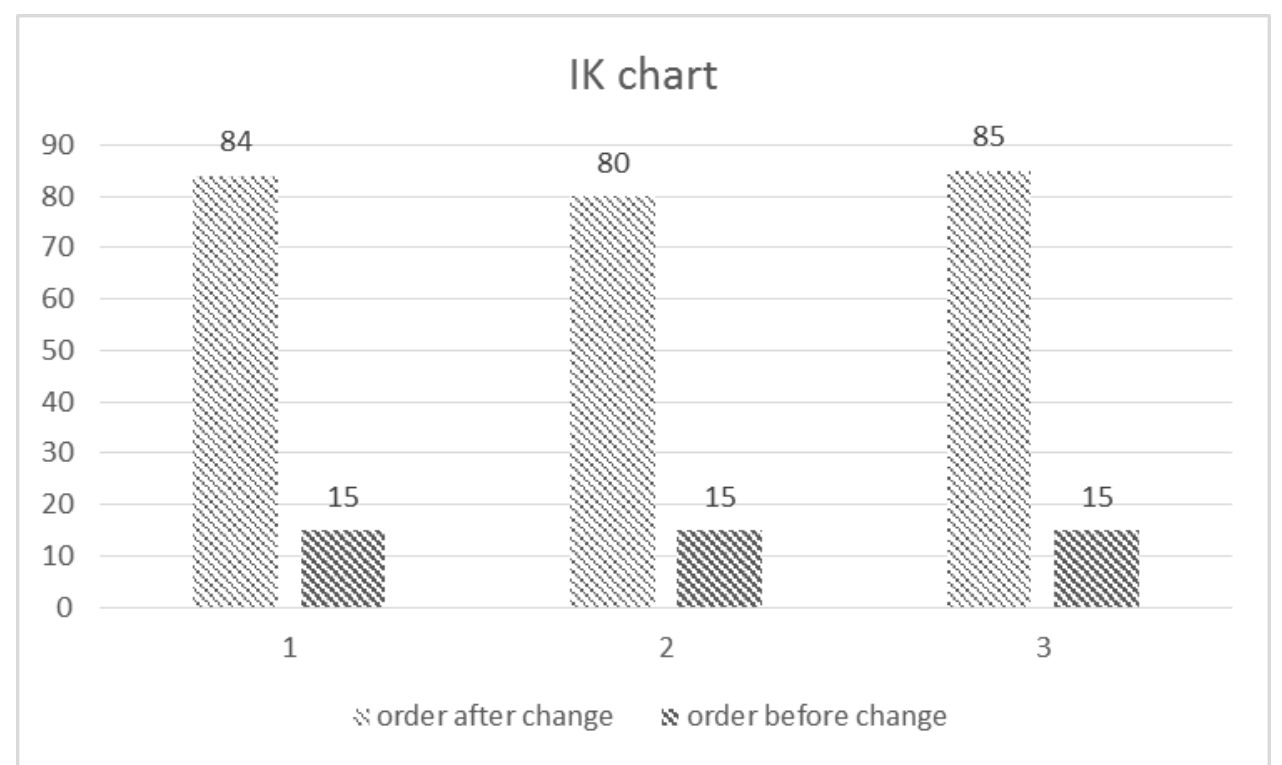

Figure 7. Comparison IK chart before and after change

From above analysis, according to the EIQ data, following optimized strategy in which both partition and batch strategy are chosen, is proposed in figure 8. 
The detailed process are in the following. All orders unified handling in three o 'clock in the afternoon in previous day, all orders are numbered in advance. Accoring to the number, partition is done by sorting requirement, then sorting operation workers in different areas handed out total number of each items cooperately, different from sorting-to-order, one more link is needed, that is, assigning the goods for each order. The advantage of this sorting strategy is that it can finishing multiple orders at the same time.

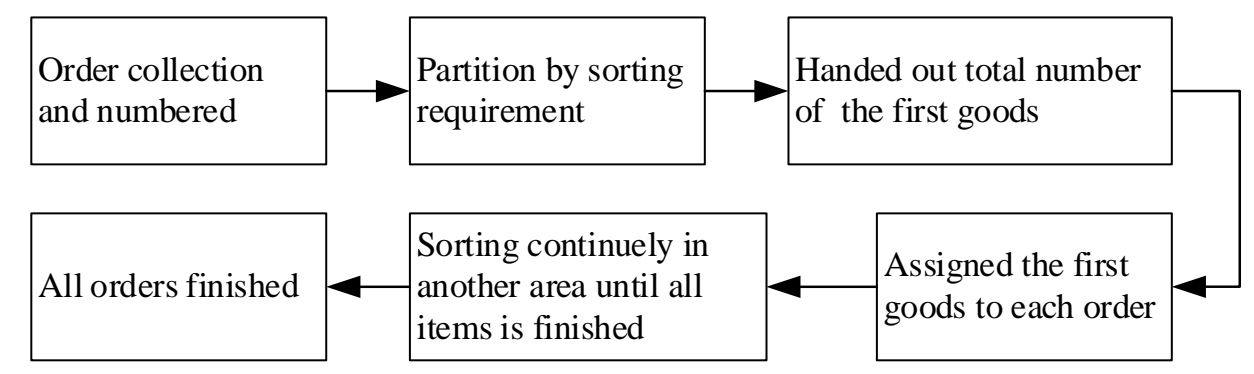

Figure 8. Optimized sorting strategy

\section{Simulation and Optimization Results}

Flexsim simulation model is a set of system design, manufacture and analysis software. It set computers‘ 3D image processing technology, simulation technology, artificial intelligence technology, data processing technology in one, dedicated to manufacturing, logistics and other fields. By using the Flexsim simulation software, the 3D model of research object can built in the computer system, and also the system analysis and engineering verification can be done, with the optimization design and retrofit scheme finally.

In this paper, generator, shelves, forklifts, synthesizers, conveyor belt, and staging areas are used for simulating sorting strategy. Generators are mainly to generate goods and pallets, shelves simulate the partition, three forklift simulate sorting operation workers, Synthesizer used to simulate the composite partition after sorting process for syntheing orders, Conveyor belt and the staging area simulate delivery process after the completion of order sorting.

Through the logistics simulation software Flexsim, simulation analysis has carried on for partition sorting and partial strategies according to the order sorting respectively, the simulation results show that proposed order partial strategy optimizes the sorting work efficiency. The comparation of sorting operation time between orginal sorting strategy and optimized sorting strategy is shown in Figure 9, which denotes that optimized sorting strategy has higher sorting efficiency about $42.86 \%$ then the orginal strategy.

\section{sorting operation time}

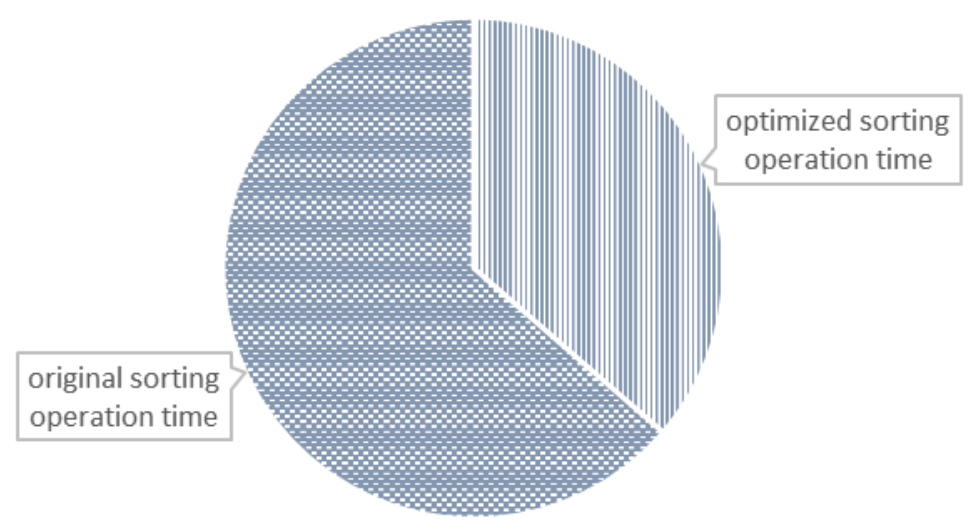

Figure 9. Comparaton sorting operation time before and after optimized 


\section{Conclusions}

Direct marketing mode of enterprise makes distribution centers has the characteristics with numerous items, small order, and frequent delivery, which increasing the operating costs of the distribution center. To remedy this problem, this study employs EIQ method to identify the characteristic of one distribution center and further analyzes the order's changes by statistic charts. Based on these analyses as well as the sorting strategy, partition strategies combined with batch sorting strategy is then formulated accordingly. With such design scheme, the sorting operation time cost of the distribution center are expected to reduce. Finally, the optimized sorting strategies is verified by Flexsim model. This paper gives a general steps and methods for sorting strategy optimization which has reference value in-operation.

\section{Acknowledgement}

This work was financially supported by the humanities and social science research project of department of education in Hei Longjiang province (1254b005), Scientific Research Fund for Doctor of Harbin University of Commerce(13DW012).

\section{References}

[1] Pushpa Rani, Sudesh Rani. New Parallel Sorting Algorithm Based on Partitioning and Redistribution. Journal of Applied Sciences, Vol. 08(2008), p.16-22

[2] Rajesh Ramachandran, E. Kirubakaran. A Comparative Study on Kakkot Sort and Other Sorting Methods. International Journal of Computer Science and Information Security, Vol.10(2010), p.16-18

[3] Shamim Akhter, M. T. Hasan. Sorting N-Elements Using Natural Order: A New Adaptive Sorting Approach. Journal of Computer Science. Vol.10(2010),p.45-55

[4] Jason Chao-Hsien Pan . A Storage Strategy for Paperless Order Picking Systems by Multi-picker. Journal of Computer Science, Vol.13(2013), p.34-52

[5] ZhaoYing. Entry-item-quantity-ABC analysis-based multitype cigarette fast sorting system. Mathematical Problems in Engineering,(2012)

[6] GaoMing, ZhouSanyuan. Research on optimization of Sorting Strategy Based on Flexsim. Logistics Technology. Vol.09(2009), p.34-36

[7] LiuTongjuan, MaXiangguo. Simulation and Optimization of Different Sorting Strategies of Distribution Centers. Logistics Technology.Vol.32(2013), p.439-444 